COMMENTARY

\title{
Innovative education and training in high power laser plasmas (PowerLaPs) for plasma physics, high power laser matter interactions and high energy density physics: experimental diagnostics and simulations
}

John Pasley ${ }^{\oplus 1}$, Georgia Andrianaki ${ }^{2}$, Andreas Baroutsos ${ }^{2}$, Dimitri Batani ${ }^{3}$, Emmanouil P. Benis $^{4}$, Andrea Ciardi ${ }^{5,6}$, Donna Cook $^{1}$, Vasilios Dimitriou ${ }^{2}$, Brendan Dromey ${ }^{7}$, Ioannis Fitilis ${ }^{2}$, Giancarlo Gatti ${ }^{8}$, Anastasios Grigoriadis $^{4}$, Marine Huault ${ }^{8,9}$, Jose Antonio Pérez Hernández ${ }^{8}$, Evaggelos Kaselouris ${ }^{2}$, Ondrej Klimo ${ }^{10}$, Michel Koenig ${ }^{5}$, George Koundourakis ${ }^{2}$, Milan Kucharik ${ }^{10}$, Jiri Limpouch ${ }^{10}$, Richard Liska ${ }^{10}$, Carlos Salgado Lopez ${ }^{8,9}$, Sophia Malko ${ }^{8,9}$, Susana Olmos-Migueláñez ${ }^{11,12}$, Yannis Orphanos $^{2}$, Valeria Ospina ${ }^{8,9}$, Nektarios A. Papadogiannis $^{2}$, Stelios Petrakis ${ }^{4}$, Jan Psikal $^{10}$, Maria Serena Rivetta ${ }^{11}$, María-José Rodríguez-Conde ${ }^{11,12}$, João Jorge Santos ${ }^{3}$, Milan Sinor ${ }^{10}$, Alexandros Skoulakis ${ }^{2}$, Ioannis Tazes ${ }^{2}$, Laura Tejada Pascual ${ }^{3}$, Calliope Tsitou ${ }^{2}$, Pavel Vachal ${ }^{10}$, Luca Volpe $^{8,13}$, Jiri Vyskocil ${ }^{10}$, Steven White ${ }^{7}$, Mark Yeung ${ }^{7}$, Ghassan Zerouli ${ }^{8,9}$, and Michael Tatarakis ${ }^{2}$

${ }^{1}$ York Plasma Institute, University of York, Heslington, York YO10 5DQ, UK

${ }^{2}$ Hellenic Mediterranean University, Institute of Plasma Physics and Lasers - IPPL, 74100 Rethymnon, 73133 Chania, Crete, Greece

${ }^{3}$ University of Bordeaux, CNRS, CEA, CELIA (Centre Lasers Intenses et Applications), UMR 5107, F-33405 Talence, France

${ }^{4}$ Department of Physics, University of Ioannina, GR 45110 Ioannina, Greece

${ }^{5}$ LULI - CNRS, CEA, Sorbonne Universiés, Ecole Polytechnique, Institut Polytechnique de Paris - F-91128 Palaiseau Cedex, France

${ }^{6}$ Observatoire de Paris, 5 Place J Janssen, Meudon 92195, France

${ }^{7}$ Centre for Plasma Physics, School of Mathematics and Physics, Queen's University Belfast, Belfast BT7 1NN, UK

${ }^{8}$ Centro de Láseres Pulsados (CLPU), Edificio M5, Parque Cientfico, C/ Adaja 8, 37185 Villamayor, Salamanca, Spain

${ }^{9}$ University of Salamanca, 37008 Salamanca, Spain

${ }^{10}$ Faculty of Nuclear Sciences and Physical Engineering, Czech Technical University in Prague, Brehova 7, 115 19, Prague 1, Czech Republic

${ }^{11}$ University Institute for Educational Sciences, University of Salamanca, 37008 Salamanca, Spain

${ }^{12}$ Group of Research “Interaction and e Learning” (GRIAL), University of Salamanca, 37008 Salamanca, Spain

${ }^{13}$ CLPU Laser-Plasma Chair at University of Salamanca, 37008 Salamanca, Spain

(Received 23 September 2019; revised 14 December 2019; accepted 13 January 2020)

Correspondence to: J. Pasley, York Plasma Institute, University of York, Heslington, York YO10 5DQ, UK. Email: john.pasley@york.ac.uk 


\begin{abstract}
The second and final year of the Erasmus Plus programme 'Innovative Education and Training in high power laser plasmas', otherwise known as PowerLaPs, is described. The PowerLaPs programme employs an innovative paradigm in that it is a multi-centre programme, where teaching takes place in five separate institutes with a range of different aims and styles of delivery. The 'in-class' time is limited to 4 weeks a year, and the programme spans 2 years. PowerLaPs aims to train students from across Europe in theoretical, applied and laboratory skills relevant to the pursuit of research in laser plasma interaction physics and inertial confinement fusion. Lectures are intermingled with laboratory sessions and continuous assessment activities. The programme, which is led by workers from the Hellenic Mediterranean University and supported by co-workers from the Queen's University Belfast, the University of Bordeaux, the Czech Technical University in Prague, Ecole Polytechnique, the University of Ioannina, the University of Salamanca and the University of York, has just finished its second and final year. Six Learning Teaching Training activities have been held at the Queen's University Belfast, the University of Bordeaux, the Czech Technical University, the University of Salamanca and the Institute of Plasma Physics and Lasers of the Hellenic Mediterranean University. The last of these institutes hosted two 2-week-long Intensive Programmes, while the activities at the other four universities were each 5 days in length. In addition, a 'Multiplier Event' was held at the University of Ioannina, which will be briefly described. In this second year, the work has concentrated on training in both experimental diagnostics and simulation techniques appropriate to the study of plasma physics, high power laser matter interactions and high energy density physics. The nature of the programme will be described in detail, and some metrics relating to the activities carried out will be presented. In particular, this paper will focus on the overall assessment of the programme.
\end{abstract}

Keywords: laser plasma interactions; postgraduate education

\section{Introduction}

'Innovative Education and Training in high power laser plasmas' (PowerLaPs) ${ }^{[1]}$ is an Erasmus Plus KA2 ${ }^{[2]}$ programme that aims to assist and enhance the studies of final-year Bachelor students, Master students and $\mathrm{PhD}$ students, improving their employability skills in the scientific area of plasma physics and high power lasers. The programme focuses on four thematic priority areas: (i) plasma physics, (ii) high power laser matter interactions and high energy density (HED) physics in theory and practice, (iii) computational modelling and simulations in laser matter interactions and (iv) plasma diagnostics. None of these areas are commonly included in university curricula, though some of the individual universities involved in the PowerLaPs programme do offer training in these areas separately ${ }^{[3-6]}$. PowerLaPs is innovative for a training course in the physical sciences in that it is an intermittent educational experience in which the students attend classes for only 4 weeks a year, yet the training is split across multiple institutes, and at each centre the teaching is delivered in a range of different ways including formal lectures, experimental laboratory sessions, computational laboratory sessions and continuous assessment (the last of these activities also continues outside the period of the workshops).

The main aim of the programme is to challenge the student participants to expand their knowledge and scientific skills in areas that are relevant to the current state-of-the-art research in the field of laser plasma interactions (LPI) and inertial fusion energy ${ }^{[7]}$. This area of expertise is relevant to a range of facilities that are either built, or being built both in Europe and internationally, for instance, the National Ignition Facility ${ }^{[8]}$, the Extreme Light Infrastructure (ELI) ${ }^{[9]}$, the CILEX-APOLLON ${ }^{[10]}$ and the European X-Ray FreeElectron Laser (XFEL) $)^{[11]}$.

Student attendees gain additional soft skills and expand their research networks by interaction with both students and academics in a range of different settings from quite formal lecture-style teaching to social settings. This encourages cross-border cooperation and enhances the professional skills of the attendees.

Throughout the programme, there are a series of assessment exercises that test the students' grasp of the material that has been delivered. These take the form of both openbook written exams carried out in a controlled environment (the results of which are discussed below) as well as longer course-work exercises, which are completed in the students' own time outside of the lectures and laboratories.

One interesting feature of the programme is that although the Learning Teaching Training (LTT) activities and two Intensive Programmes (IPs) that form the basis of the PowerLaPs are constructed as a single educational course, students are not required to attend all of the sessions. Students receive individual certification for attendance at each of the LTTs/IPs, and they can opt to attend from one to all of the events. For this reason, the individually taught modules have to be carefully designed to enable the participation of students who have not necessarily attended previous workshops. As shown in Ref. [12], the course has been successful in this respect, with students who had not attended previous training events faring similarly well in examinations to their counterparts who had.

The material developed as part of the PowerLaPs programme will be made available worldwide through the PowerLaPs website, which was set up partially in parallel to the evolution of the programme $e^{[1]}$. At present, and until the completion of the programme, these resources are only 
available to students from the participating institutes via the e-Class platform hosted by the Hellenic Mediterranean University, Institute of Plasma Physics and Lasers (IPPL) ${ }^{[13]}$.

\section{Subject areas}

The different topics covered by the PowerLaPs programme are divided into four areas of specialization labelled O1, O2, $\mathrm{O} 3$ and $\mathrm{O} 4$. Each of these is described below.

\subsection{O1 Plasma Physics - Theory and Experiments}

This is the theoretical and experimental part of the Plasma Physics thematic priority educational material. The LTT in this area took place at the University of Bordeaux in January 2018, with further education in this area taking place at the first IP that was held at the Hellenic Mediterranean University, IPPL in July 2018. The delivery of this material is described in detail in Ref. [12].

\subsection{O2 High Power Laser Matter Interactions/High Energy Density Physics - Theory and Experiments}

This is the theoretical and experimental part of the High Power Laser Matter Interactions/High Energy Density Physics material. The LTT in this area took place at Queen's University Belfast in March 2018, with further education in this area taking place at the first IP that was held at the Hellenic Mediterranean University, IPPL in July 2018. The delivery of this material is described in detail in Ref. [12].

\subsection{O3 Computational Modelling and Simulations in Laser Matter Interactions}

This is the Computational Modelling and Simulations in Laser Matter Interactions material. The LTT in this area took place at the Prague Technical University in January 2019, followed by further training at the second IP in July 2019 at the Hellenic Mediterranean University, IPPL. The LTT in Prague included the following topics, delivered in lecture-style format: (1) Fundamentals of Numerical Methods, (2) Solving Partial Differential Equations (PDEs), (3) Simulations of Laser-Produced Plasmas (LPP), (4) Particle-In-Cell (PIC) Simulations of Laser Plasma Interactions (LPI), (5) Fluid Simulations of LPP, (6) Monte Carlo Simulations for LPI, (7) Description of HED Matter, (8) Atomic Physics of Multiply Ionized Plasmas and (9) Parallel Algorithms for LPP Simulations; and in addition, the following laboratory sessions: (1) Numerical Methods, (2) PIC Simulations of LPI, (3) Fluid Simulations of LPP and (4) Monte Carlo Simulations for LPP.

\subsection{O4 Laser Plasma Diagnostics - Theory and Experiments}

This is the Laser Plasma Diagnostics related educational material. The LTT in this area took place at the University of Salamanca in March 2019, followed by further training at the second IP. The LTT in Salamanca included the following topics, delivered in lecture-style format: (1) Introduction to Laser-Plasma Diagnostics, (2) Characterization of High Intensity Lasers, (3) Photonic-Based Electron Density Diagnostics, (4) K-Alpha Measurement of Proton Beams, (5) Optical Streak Camera Methods, (6) Numerical Methods for Diagnostics, (7) Introduction to Solid-State Lasers, (8) Terahertz Detection and Emission Using PlasmaWave-Based Devices, (9) Ultrafast Laser Pulse Characterization and Applications, (10) Charged Particle Transport in Warm Dense Matter and HED Plasma, (11) UnderCritical and Near-Critical Density Plasmas: Laser-Driven Particle Acceleration and Diagnostics, (12) Charged Particle Detectors - the Thompson Parabola, (13) Liquid Targets, (14) X-ray Diagnostics - the Kirk Patrick-Baez Microscope, (15) Charged Particle Detectors - Time of Flight Techniques and (16) Radiation Protection in Short Duration Events; and in addition, the following laboratory sessions: (1) Terahertz Generation, (2) Liquid Target Interferometry, (3) X-ray Generation and Diagnostics and (4) Oscillators.

The second IP, held at IPPL in Crete, spanned both O3 and $\mathrm{O} 4$. The lectures and laboratories held at the IP that are relevant to these two topics are therefore listed together. The following topics were delivered in a lecture-style format: (1) Plasma Kinetics - PDEs for Plasma and Particles, (2) PIC Simulations of LPI, (3) Introduction to Laser-Plasma Process Diagnostics, (4) Advanced PIC Simulations and their Applications, (5) Diagnostics for Ions and Ions as Diagnostics, (6) Fokker-Planck and Fluid Simulations of LPI, (7) Shock Dynamics and Diagnostics, (8) Fluid Simulations - Atomic Physics and Radiation Transport, (9) Diagnostics for Inertial Fusion, (10) Atomic Physics in Plasma Diagnostics, (11) High Harmonic Generation as a Diagnostic for Laser Contrast Using Simulations and Experiments, (12) Novel Streaking Experiments for Studying Ultrafast Proton Bursts from LPP, (13) Ultrafast Laser Diagnostics, (14) Multiphysics Simulations for LPP, (15) Ultrafast Bunch Duration Measurements through Optical Diagnostics, (16) Diagnosing and Simulating Hydrodynamic Behaviour in High Intensity Short Pulse LPI and (17) Astrophysics with High Power Lasers; and in addition, the following laboratory sessions: (1) Laser Matter Interaction Diagnostics for a Terawatt Ultrafast Laser System, (2) Plasma Simulations - Magnetohydrodynamic Simulations, (3) Laser Matter Interactions - Finite Element Method (FEM) Simulations, (4) Laser Matter Interaction Diagnostics, (5) Plasma Pinch Diagnostics, (6) LaserSolid Target Interaction - FEM Simulations and (7) PIC Simulations of Particle Acceleration. 


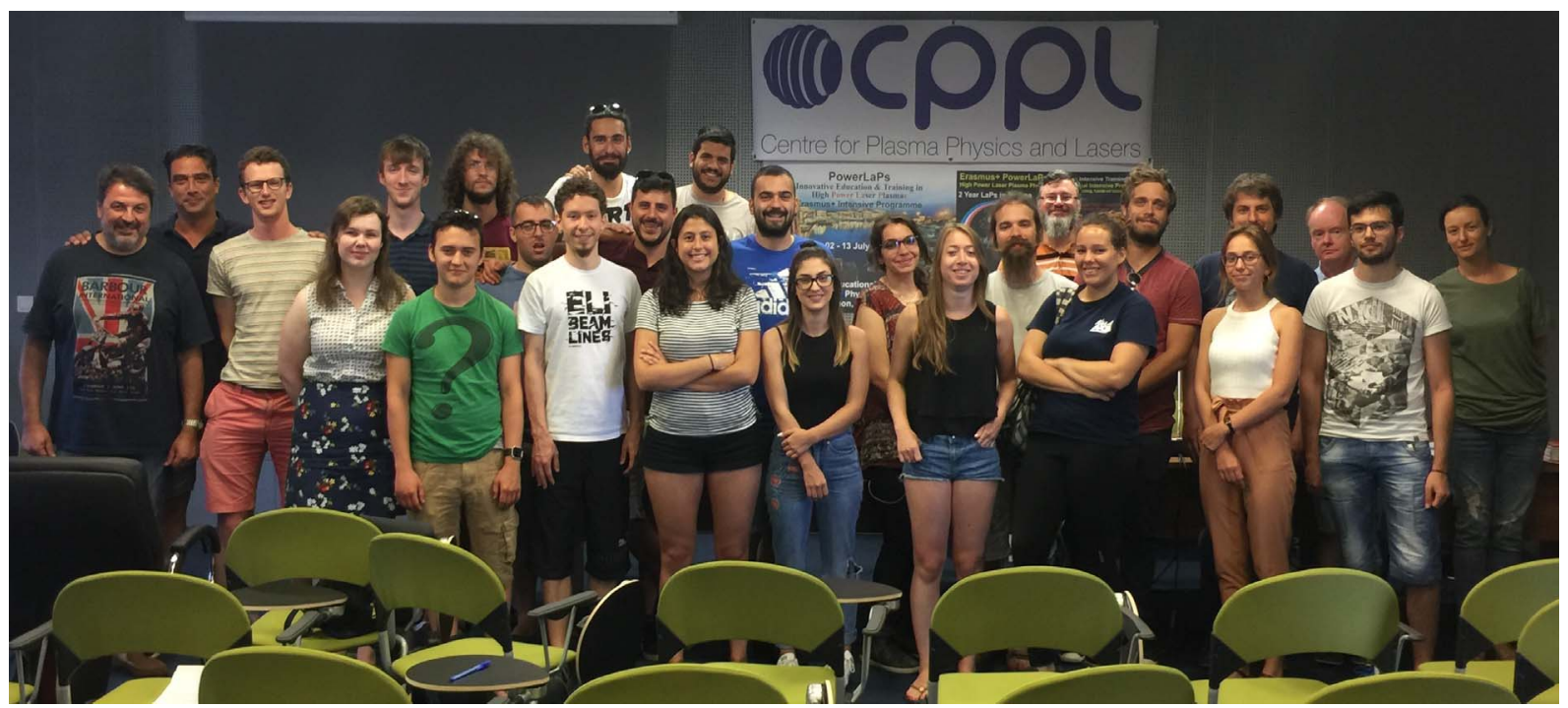

Figure 1. Attendees at the first IP in Crete.

\section{Participation and usage statistics}

All of the participating institutes have taken good advantage of the resources offered by the PowerLaPs programme. More detailed figures are provided below both for LTT attendance and e-Class platform usage.

\subsection{LTT attendance}

All six of the LTTs conducted to date have been well attended. Nonlocal institutes received funding from the programme to send three students to each of the LTTs/IPs, meaning that the number of visiting students who can be funded to each activity is 21 , with the home institute sometimes having a larger number of students participate. Attendance figures for the first year of PowerLaPs are given in Ref. [12]. In the second year, the LTT at Prague was attended by 22 students, the LTT at Salamanca was attended by 22 students and the second IP in Crete was attended by 30 students. This means that in every case, more students attended the LTTs than could be expected based on the funding available. A photograph of some of the attendees of the first IP is shown in Figure 1.

\section{2. e-Class platform usage}

The e-Class platform (which is currently only accessible to teachers, tutors and students from the partner universities) has been used by all of the students who have participated in the programme in addition to some others from the participating institutes. The material related to $\mathrm{O} 3$ has thus far been visited by 126 participants, of whom 19 were academics and the remaining 107 students. The total number of visits made to the site by these users was 470 . The educational material related to $\mathrm{O} 4$ has received 126 visitors, of whom (again) 107 were students and the remainding academics. The total number of site visits by these users was 347. In common with the $\mathrm{O} 1$ and $\mathrm{O} 2$ usage data that are reported in Ref. [12], it is once again noteworthy that the total number of students to be formally involved in at least one of the LTTs of the PowerLaPs programme to date is significantly less than the total number of students who accessed educational material on the e-Class platform. Eighty-five students were formally involved in at least one of the LTTs held to date. This suggests that around 33\% of the students who have used the e-Class platform were using it independent of formal participation in the PowerLaPs programme.

\section{Student performance}

In the Prague LTT, the average mark attained across the cohort of 22 student attendees was $60.00 \%$ with a standard deviation of 11.41. In the Salamanca LTT, the average mark attained by a different set of 22 student attendees was $68.88 \%$ with a standard deviation of 12.48 . In the second IP in Crete, the average mark among the 30 student attendees was $63.48 \%$ with a standard deviation of 8.82 . There are no strong trends to discuss here, and the interested reader is referred to Ref. [12] in which the student performance in the first three LTTs was discussed in some detail. 
Table 1. Quantitative performance metrics for each of the LTTs in the PowerLaPs programme.

\begin{tabular}{|c|c|c|c|c|c|c|c|c|}
\hline Metric & O1 LTT & O2 LTT & IP1 & 03 LTT & 04 LTT & IP2 & Mean & Expert \\
\hline All actions were performed on time by all partners & 10 & 10 & 10 & 10 & 10 & 10 & 10 & 10 \\
\hline Efficiency of the structure of teaching modules & 10 & 10 & 10 & 10 & 10 & 10 & 10 & 10 \\
\hline Mean value of student's performance in assessments & 6.4 & 7.7 & 7.2 & 6.0 & 6.8 & 6.4 & 6.8 & 7 \\
\hline Quality and quantity progress on deliverables & 9 & 8 & 9 & 9 & 9 & 9 & 8.8 & 9 \\
\hline Number of attendants per partner & 9.2 & 9.6 & 10.0 & 9.2 & 8.3 & 9.1 & 9.2 & 9 \\
\hline Visits, hits and downloads on the e-Class and PowerLaPs site & 9 & 8 & 9 & 9 & 9 & 9 & 8.8 & 10 \\
\hline Mean value from questionnaires of participants & 8.5 & 8.6 & 9.0 & 8.4 & 8.8 & 8.9 & 8.7 & 9 \\
\hline Mean & 8.9 & 8.8 & 9.2 & 8.8 & 8.8 & 8.8 & 8.9 & 9.1 \\
\hline
\end{tabular}

\section{Multiplier event}

In August 2019, a Multiplier Event was held at the University of Ioannina, with meetings and lectures spanning 2 days. On the first day, principal investigators (PIs) from the institutes involved in PowerLaPs met to formally discuss and summarize the PowerLaPs programme. On the second day, the PIs gave a series of talks and lectures to their colleagues, assembled undergraduate and graduate students from the University of Ioannina and other Greek institutes, attendees from the private sector and an external observer and expert in the field of high power laser plasma physics. Topics included a summary of the PowerLaPs programme, a summary of the evaluation and performance of the programme and a set of five physics lectures describing core elements of the programme for the student members of the audience. The total number of student attendees at this 1-day event was 61, the majority of these coming from the University of Ioannina (53).

\section{Dissemination}

PowerLaPs outputs have been assembled into an e-Class platform that is open to partner institutions, which will be later made accessible worldwide. The e-Class platform provides access to all of the digital educational and training material that has been delivered as part of the programme.

The University of Ioannina has built and is administrating the web page of the programme ${ }^{[1]}$, in which the actions and final deliverables of each year are uploaded and further disseminated to the scientific community. The structure of the uploaded educational material allows for interactive lifelong learning and teaching.

The Hellenic Mediterranean University is taking the lead in publicizing the programme using social media. This and our previous paper (Ref. [12]) will also serve to disseminate some information about PowerLaPs to colleagues within the higher education sector.

\section{Programme evaluation}

The success of each of the six LTTs is assessed in terms of seven metrics: (1) whether all actions were performed on time by all partners, (2) the efficiency of the structure of teaching modules in order to result in the proper outcomes, (3) the mean value of student's performance in assessments, (4) quality and quantity progress of deliverables, (5) the number of attendants per partner, (6) the number of visits, hits and downloads on the e-Class and PowerLaPs site and (7) the mean value from questionnaires of participants (described in more detail below). Each of these metrics is scored numerically out of 10 , to two significant figures. These results and programme averages are presented in Table 1.

The external expert in making his final assessment of the programme also grades its success in terms of the following three metrics: (1) the successful accomplishment of the project, (2) the number of attendants influenced and of beneficiaries of PowerLaPs and (3) the overall quality and quantity, the progress of the deliverables' completeness during the lifespan of the project and the final intellectual outputs of O1-O4. Each of these metrics is also scored numerically out of 10 , to two significant figures. The programme was awarded 10 for each of these three metrics by the external expert, indicating that it was a success.

\subsection{Questionnaires}

As part of the assessment of the performance of the programme, both teachers and students who were involved in the PowerLaPs LTTs were asked to complete questionnaires in order to relate their level of satisfaction with the content and delivery of the programme material. These questionnaires were broken down into eight distinct sections, each with a set of relevant questions scored on an integer rating scale of $1-5$, with 5 representing the best case. The eight different sections of the questionnaire along with the mean scores across all six LTTs were: (1) Objectives of the study plan (4.37), (2) Planning of the teaching (4.40), (3) Development of teaching and learning evaluation (4.30), (4) Student 
admission (4.31), (5) Student guidance (4.32), (6) Academic infrastructure (4.49), (7) Teacher evaluation (4.4) and (8) Training results (4.56). There was little variation among the different LTTs as shown in the questionnaire-based metric in Table 1.

In addition, we collected some comments from the students, a few of which are given below anonymously.

The schools provided a great opportunity to network with other students and academics in the laser-plasma field. It also introduced me to facilities I had not heard of yet and opened up future collaboration options.

A constructive and rewarding experience, helping us to develop the skills and knowledge we need in this dynamic and innovative field.

The training schools provided a broad spectrum of lectures which meant you engaged with a larger field of work that normal. This meant the understanding was improved in a range of fields rather than on a single narrow topic. As someone who primarily produces simulations, the chance to get lectures from experimental experts was of great benefit in understanding how experimentalists work and the results experiments can produce. The school provided the opportunity to engage with students and academics from across Europe for a prolonged period of time, which was just as engaging culturally as it was academically.

The PowerLaPs training school provided an excellent assortment of teaching materials and hands-on sessions, while offering an exciting opportunity to meet a wider international community of fellow postgraduate laser-physics students.

PowerLaPs has given me a good balance of theory and numerical training in all aspects of laser-plasma research with the opportunity to apply what I learned in hands-on experiments. It has also been a great networking opportunity for me, giving me the chance to meet and work with a number of people across a wide range of facilities.

The PowerLaPs training school was very beneficial in connecting me to academics and other students across Europe. The experimental courses were particularly useful and the content has been applied to experiments relevant to the $\mathrm{PhD}$ topic.

\subsection{Summary statement of the external expert observer}

The expert evaluation of the PowerLaPs programme was performed by Prof. George Throumoulopoulos from the Department of Physics at the University of Ioannina. $\mathrm{He}$ writes:

All the above actions were properly advertised and disseminated by the partner institutions either on the web pages of the institutes and/or the official web page of the programme https://powerlaps.chania.teicrete.gr/ as well as other events. Each partner organization proposed and sent a maximum of three students as a sending partner to visit the various LTTs. Only in a few cases were three students not able to be sent by each partner. The lectures and the supporting educational documents of the LTTs were uploaded on time from the tutors in the form of documents, manuals, presentations and tutorials to the e-Class platform. Students were registered to the e-Class platform and gained early access to the educational material providing them with time to get prepared for the LTTs. Attendants had a short exam session on the fifth day of the shorter LTTs and received further instructions from the tutors for implementing their continuous assessment exercises, and more generally studying plasma physics, in the runup to the IPs in Crete. For the two IPs held at Rethymnon in Crete, up to one teacher from each partner organization travelled to Crete to lecture. A complete educational course based on the intellectual outputs was offered.

The dissemination of the programme was pursued by the following actions: (1) 'traditional' dissemination actions involving two publications in relevant journals, state of the art reports, publications in popular public journals for science, newspapers, etc., (2) 'modern' dissemination actions involving electronic tools: Facebook, advertisement via web pages, etc., (3) e-educational, e-training, e-learning, and etutoring material of PowerLaPs being released on the website, and (4) a multiplier event.

The expert's overall evaluation scores are presented in the final column of figure Table 1.

\section{Conclusions}

PowerLaPs is a novel programme of short LTTs and IPs, combining a multi-centre approach with a range of different delivery formats. Six LTTs, including two IPs, have been carried out, covering the topics of (i) plasma physics, (ii) high power laser matter interactions and HED physics, 
(iii) computational modelling and simulations in laser matter interactions and (iv) laser plasma diagnostics. All six events have been well attended with a total of 85 students having formally attended at least one of the six events. Student performance has been within the expected range.

\section{Acknowledgements}

The authors acknowledge the financial support of the Erasmus Plus and the IKY/ Erasmus+ Hellenic National Agency. They acknowledge the support of the administrative teams of the universities involved in PowerLaPs. They also acknowledge the support by computational time granted from the Greek Research and Technology Network (GRNET) in the National HPC facility ARIS under project ID pr007020 LaMIPlaS-II. The IPPL, access point of the National Research Infrastructure (HELLAS-CH, HiPER-ELI-LaserLab Europe and Iperion $\mathrm{CH}$ ), acknowledges the support by 'ELILASERLAB Europe Synergy, HiPER and IPERION-CH.gr' (MIS 5002735), which is implemented under the Action 'Reinforcement of the Research and Innovation Infrastructure', funded by the Operational Programme 'Competitiveness, Entrepreneurship and Innovation' (NSRF 2014-2020) and co-financed by Greece and the European Union (European Regional Development Fund).

\section{References}

1. PowerLaPs website: https://powerlaps.chania.teicrete.gr/.
2. Erasmus Plus website: http://ec.europa.eu/programmes/erasm us-plus/.

3. University of York Fusion MSc website: https://www.york.ac .uk/study/postgraduate-taught/courses/msc-fusion-energy/.

4. French Msc consortium FedSPF in Plasmas and Fusion Science website: http://www.master-plasmas-fusion.fr/?lang= en.

5. Technological Educational Institute of Crete MSc in Laser Plasma and Applications website: http://www.cppl.teicrete.g r/lapla-msc-degree.

6. MSc in Physics and Technology of Thermonuclear Fusion of the Czech Technical University in Prague website: https://ww w.fjfi.cvut.cz/en/education/master-s-study/fields-of-master-sstudy/ing-physics-and-thermonuclear-fusion-technology.

7. Collection of Special Topic papers on inertial confinement fusion (Special Issue), Nuclear Fusion, Volume 54, Issue 5 (2014).

8. National Ignition Facility website: https://lasers.llnl.gov/.

9. Extreme Light Infrastructure website: https://eli-laser.eu/.

10. CILEX-APOLLON website: https://portail.polytechnique.edu /luli/en/cilex-apollon/apollon.

11. The European X-Ray Free-Electron Laser website: https://ww w.xfel.eu/.

12. J. Pasley, G. Andrianaki, A. Baroutsos, D. Batani, E. P. Benis, M. Borghesi, E. Clark, D. Cook, E. D’Humieres, V. Dimitriou, B. Dromey, M. Ehret, I. Fitilis, A. Grigoriadis, S. Kar, E. Kaselouris, O. Klimo, M. Koenig, K. Kosma, G. Koundourakis, M. Kucharik, A. Lavery, J. Limpouch, Y. Orphanos, N. A. Papadogiannis, S. Petrakis, D. Riley, M. S. Rivetta, L. T. Pascual, J. J. Santos, A. Skoulakis, I. Tazes, V. Tikhonchuk, J. Trela, C. Tsitou, L. Volpe, S. White, M. Yeung, and M. Tatarakis, High Power Laser Science and Engineering 7, e23 (2019).

13. Institute of Plasma Physics and Lasers - IPPL, Hellenic Mediterranean University website: http://www.ippl.hmu.gr/. 